\title{
Shampoo Dosage Form
}

National Cancer Institute

\section{Source}

National Cancer Institute. Shampoo Dosage Form. NCI Thesaurus. Code C42981.

A solution or suspension composed of an anionic surfactant and intended for administration to the hair and/or scalp. 